\title{
Isolation and Characterization of Endophytic Bacteria in Soybean (Glycine max L. (Merrill) Cultivated on Alluvial Soil of Can Tho city, Vietnam
}

\author{
Van Thi Phuong Nhu \\ Dept. of Biology Science, Phu Yen University, Vietnam \\ Cao Ngoc Diep \\ Dept. Microbiology Biotechnology, Biotechnology R\&D Institute, \\ Can Tho University, Can Tho City, Vietnam
}

\begin{abstract}
Eighty-six endophytic bacterial isolates were isolated from 70 soybean plants (including whole plant as stem, root and nodule) which collected at 5 districts of Can Tho city, Mekong Delta, Vietnam; they developed on three kinds of medium (PDA, TSA and G6) after 2 or 3 days incubation and they made the pellicles on semi-solid media. The bacterial isolates were tested in-vitro for plant growth promoting properties including nitrogen fixation, phosphate solubilization and IAA production together with producing siderophores. All of them had the ability of ammonium synthesis, phosphate solubilization and IAA biosynthesis but there were $38.37 \%$ bacterial isolates producing siderophores. The sequences from selected nitrogen-fixing and phosphate-solubilizing bacteria (26 isolates) showed high degrees of similarity to those of the GenBank reference strains (between $97 \%$ and $100 \%$ ). From 26 isolates, 7 belonged to Bacilli and 17 were Gamma-Proteobacteria. Based on Pi value (nucleotide diversity), Bacilli group had the highest Theta value and Thete values (per sequence) from S of SNP for DNA polymorphism were calculated from each group and Bacilli group had the highest values in comparison with gammaproteobacteria. From these results showed that there are five strains as Enterobacter cloacae TSR1A, Enterobacter cloacae CPR1A, Bacillus sp. OSR12, Bacillus subtilis TST10c and Acinetobacter sp. TGN1 revealed promising candidates with multiple beneficial characteristics and they have the potential for application as inoculants adapted to unfertile soil and local crops because they are not only best strains but also combined with rhizobia strains for improvement of better grain yield and quality seed of soybean cultivation on alluvilal soil in the future.
\end{abstract}

Keywords: 16S rRNA Gene Sequence, Alluvial soil, Endophytic Bacteria, Stem, Root, Root-nodule of soybean

\section{INTRODUCTION}

Soybean (Glycine max) is one of the most important oil seed crop in the world. It contains 18 to $22 \%$ oil, highly desirable in diet and have 40 to $42 \%$ of good quality protein [1]; Soybean protein is rich in valuable amino acid lycine (5\%) in which most of the cereals are deficient [2]. Soybean (Glycine max (L.) Merrill) is an Asiatic leguminous plant, occupying large acreages of land worldwide for its oil and protein [3]. Rhizobia are perhaps the best known beneficial plant-associated bacteria because of the importance of the nitrogen fixation that occurs during the Rhizobium-legume symbiosis [4]. In recent years, interest in endophytic micro-organisms has increased, as they play a key role in agricultural environment and are promising because of their potential use in sustainable agriculture [5]. Endophytes have been found in almost every plant studied [6]; endophytes are sheltered from environmental stresses and microbial competition by the host plant, and they seem to be ibiquitous in plant tissues, having been isolated from flowers, fruits, leaves, stems, roots, and seeds of various plant species [7]. Endophyte-plant associations have been found to improve plant health and may help host plant to rescue from various biotic and abiotic stresses [8][9]. Endophytic bacteria have been isolated from legume plants such as alfalfa [10], clover [11], pea [12] and soybean [13]. Besides that, Sturz et al. [11] reported the 
isolation of 15 non-rhizobial species from clover root nodules, eight of which were found only in root nodule tissues.

Can Tho city locates in the center of Mekong Delta, Vietnam (10 01'57' N and $\left.105^{\circ} 47^{\prime} 03^{\prime \prime} \mathrm{E}\right)$ (Figure 1), composes of 5 districts and 4 towns with $1409 \mathrm{~km}^{2}$ (over $80 \%$ agriculatural land) (GENERAL STATISTICS OFFICE of VIET NAM, 2016). Together with corn, mung bean, sesame seeds and other crops, soybean has been cultivated routinely on alluvial soil in dry season each year.

This study was aimed to isolate the non-nodulating endophytic bacteria from the root nodules and soybean plants (stem and root). Using 16S rRNA gene sequence analysis, we also studied the taxonomic position of these non-nodulating endophytic bacteria and compared endophytic bacterial isolates which planted in alluvilal soil in the Mekong Delta

\section{MATERIALS AND METHODS}

\section{A. Plant sample and Isolation endophytic bacteria in soybean nodules and plants}

Soybean plants used in the experiment were local cultivars (Glycine max L. Merr), were cultivated at 5 districts as Vinh Thanh, Co Do, Thoi Lai, Thot Not, O Mon in Can Tho city (Figure 1). Thot Not, and O Mon are districts which locate along the Hau river.
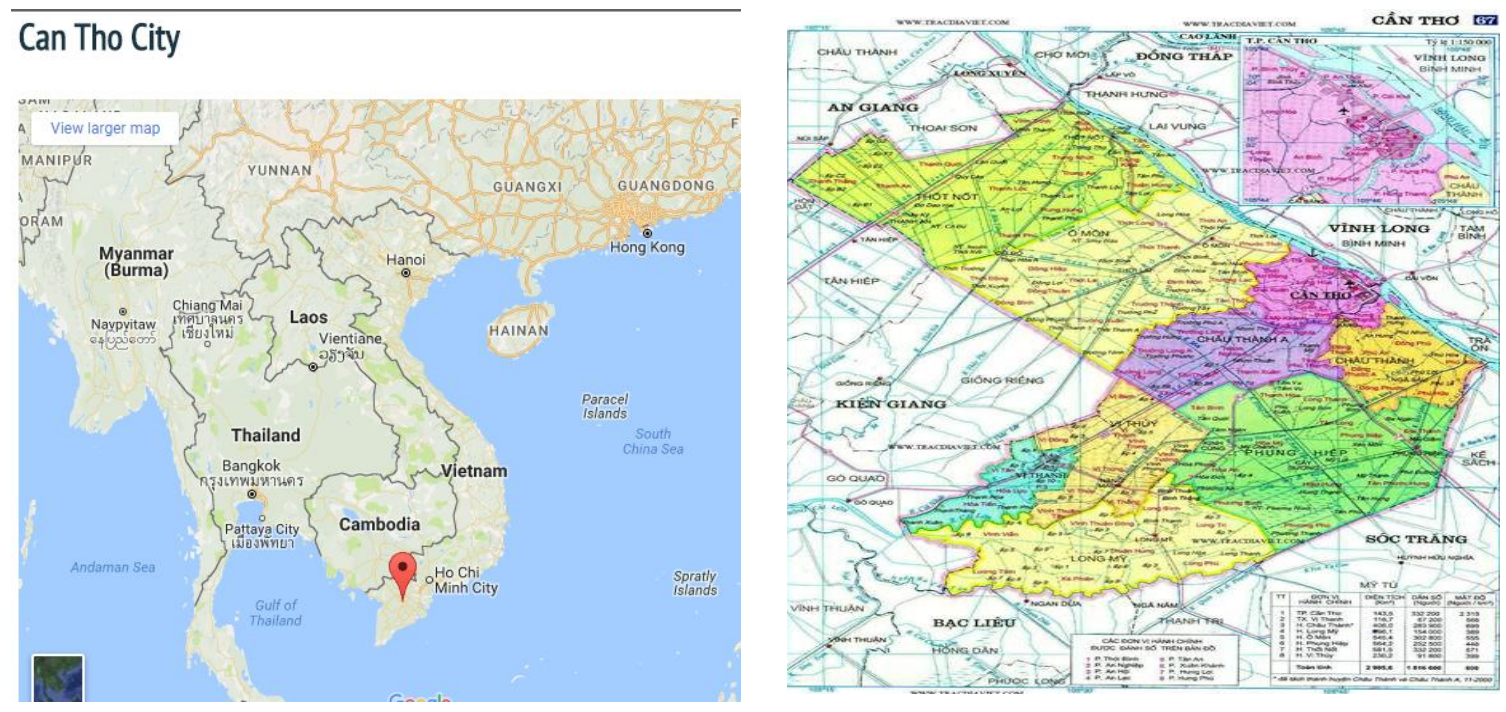

Figure 1. Can Tho city map

Plant samples were collected at the flowering-stage (35-40 days after sowing), five samples were carefully removed, washed under tap water to remove soil, and separated into roots and nodules. Nodules were put in beaker, soaked in distilled water, and drained. They were rinsed in $70 \%$ ethanol for $30 \mathrm{~s}$ and then sterilized with $0.1 \% \mathrm{HgCl}_{2}$ for $3 \mathrm{~min}$ [4]. After that, nodules were washed ten times with sterile water [10]. Surface-disinfected tissue was aseptically macerated with homogenizers and tissues were diluted with $1 \mathrm{~mL}$ sterile water. One hundred microliters from appropriate dilutions were palted on two different media, viz potato dextrose agar (PDA) and tryptic soy agar (TSA) [14] together with G6 medium [glycerol instead of mannitol] [15].

Samples were obtained whole plant after that soil rhizosphere was separated for described experiments above, soybean roots and stems were washed with tap water to remove attached clay; stems and roots were cut separately. Subsequently, the stems and roots were immersed in $70 \%$ ethanol in 3 min, washed with fresh sodium hypochlorite solution $\left(2.5 \%\right.$ available $\left.\mathrm{Cl}^{-}\right)$for $5 \mathrm{~min}$, rinsed with hydrogen peroxide (3\%) for 30s and finally 
washed five times with sterile distilled water. To confirm that the sterilization process was successful, the aliquots of the sterile distilled water used in the final rinse were set on tryptone - yeast extract - glucose agar medium plates. The plates were examined for bacterial growth after incubation at $28^{\circ} \mathrm{C}$ for 3 days. Soybean stem and root samples that were not contaminated as detected by culture-dependent sterility test were used for further analysis. Samples (stems or roots) were cut to $1-2 \mathrm{~cm}$ pieces and macerated with a sterile mortar and pestle; tissue extracts were then serially diluted 10-fold in sterile water, $200 \mu$ l-aliquot samples were used to inoculate in (in triplicate) Nitrogen-free semisolid LGI in $5 \mathrm{ml}$ tubes. After 48-72 $\mathrm{h}$ incubation, bacteria growing in tubes as a white or yellow pellicle at a depth of 1 to $4 \mathrm{~mm}$ were streaked on LGI agar plates, the cultures were streaked on media to obtain single colonies.

Bacterial colonies were differentiated from the basis of colony morphology and pigmentation. The colonies were subculture on the agar-based subculture medium plates by striking technique and re-incubated at $30^{\circ} \mathrm{C}$ for 4 days. This isolation process carries out in shifts of the agar-based culture medium to the agar-based subculture medium until monocultures were obtained. Monocultures were culture on the agar-based culture medium slant in the test-tube $(12 \mathrm{ml})$ and incubated at $30^{\circ} \mathrm{C}$ for 4 days following by stored $4^{\circ} \mathrm{C}$ in refrigerator.

Morphological characterization of the isolates was carried by Gram staining. For motility, each isolate was spot-inoculated on the center of semi-soloid nutrient agar plates $(0.2 \%$ agar $)$ and incubated at $30^{\circ} \mathrm{C}$ [4]. Cell shape was observed under light microscope, colony characterization as size, color, shape were recorded at $2-3$ days after plating into petri-dishes.

\section{B. Characterization of endophytic bacteria for plant growth promoting attributes}

Bacterial isolates were also studied in vitro for plant growth promoting properties including indole acetic acid (IAA) production, nitrogen fixation, solubilization of phosphate.

For indoleacetic acid production, $5 \mu \mathrm{l}$ for $\log$ phase culture was inoculated in $5 \mathrm{ml}$ of LB (Luria-Bertani; Bacto-Tryptone $10.0 \mathrm{~g} / \mathrm{l}$, yeast extract $5.0 \mathrm{~g} / \mathrm{l}, \mathrm{NaCl} 5.0 \mathrm{~g} / \mathrm{l}$ ) broth with L-tryptophane and incubated on shaker for $24 \mathrm{~h}$. Auxin quantification was carried out following the method of Gordon and Weber [16].

For nitrogen fixation ability and phosphate solubilization: the ability to fix $\mathrm{N}_{2}$ was tested on Burk' $\mathrm{N}$ free liquid medium incubation at $30^{\circ} \mathrm{C}$ and the ammonium concentration in medium was measured by Phenol Nitroprusside method after 2,4,6 and 8 day inoculation (DAI) and inorganic phosphate solubilization ability was tested on NBRIP liquid medium and they incubated at $30^{\circ} \mathrm{C}$ and the $\mathrm{P}_{2} \mathrm{O}_{5}$ concentration was measured by ammonium molypdate method after 5, 10, 15 and 20 DAI [17]. Furthermore, siderophore production was assayed by the bacterial isolates according to Schwyn and Neilands [18] using NBRIP medium without tryptophan which was diluted fivefold. The isolates were inoculated spot onto Chrome azurol S agar plates divided into equal sectors, and the plates were incubated at $28^{\circ} \mathrm{C}$ for $48 \mathrm{~h}$. Development of a yellow, orange or violet halo around the bacterial colony was considered to be positive for siderophore production.

\section{C. $16 S$ rDNA gene amplification and sequencing}

Bacterial DNA isolated was conducted by published protocols [19] and the following primers were used for PCR amplification of $16 \mathrm{~S}$ ribosomal DNA: p515FPL [20] and p13B [21] [22]. The 50 $\mu \mathrm{L}$ reaction mixture consisted of $2.5 \mathrm{U}$ Taq Polymerase (Fermentas), $0.1 \mathrm{mM}$ of each desoxynecleotide triphosphate, $1.5 \mathrm{mM}$ magnesium chloride, $0.4 \mathrm{mM}$ spermidine (Sigma), $10 \mathrm{pM}$ of each primer (Fermentas) and $10 \mathrm{ng}$ DNA, 10\% ( vol/vol) dimethyl disulfide (Fermentas). The thermocycling profide was carried out with an initial denaturation at $94^{\circ} \mathrm{C}(3 \mathrm{~min})$ followed by 30 cycles of denaturation at $94^{\circ} \mathrm{C}(60 \mathrm{~s})$, annealing at $57^{\circ} \mathrm{C}(60 \mathrm{~s})$, extension at $72^{\circ} \mathrm{C}$ $(120 \mathrm{~s})$ and a final extension at $72^{\circ} \mathrm{C}(4 \mathrm{~min})$ in $\mathrm{C} 1000$ Thermal Cycler (Bio-Rad). Aliquots $(10 \mu \mathrm{l})$ of PCR products were electrophoresed and visualized in $1 \%$ agarose gels using standard electrophoresis procedures. Partial 16S rRNA gene of selectived isolates in each site was sequenced by MACROGEN, Republic of Korea (dna.macrogen.com). Finally, 16S rRNA sequence of the isolate was compared with that of other microorganisms by way BLAST (http://www.ncbi.nlm.nih.gov/BLAST/Blast.cgi); In the best isolate(s)(high ability of nitrogen fixation, phosphate solubilization and IAA synthesis) and 16 isolates of 3 sites were chosen to sequence and the results were compared to sequences of GenBank based on partial 16S rRNA sequences to show relationships between endophytic strains [23] and phylogenetic tree were constructed by the neighbor-joining method using the MEGA software version 6.06 based on 1000 bootstraps. 


\section{SNPS Discovery}

The sequence date from 17 root-associated bacterial isolates were analysed with SeqScape@Software (Applied Biosystem, Foster City, CA, USA). SeqScape is a sequence comparison tool for variant identification, SNP discovery and validation. It considers alignment depth, the base calls in each of the sequnces and the associated base quality values. Putative SNPs were accepted as true sequence variants if the quality value exceeded 20. It means a $1 \%$ chance basecall is incorrect.

\section{E.Nucleotide Diversity $(\theta)$}

Nucleotide diversity $(\Theta)$ was calculated by the method described by Halushka et al. [24]

$$
\Theta=\mathrm{K} / \mathrm{aL} \quad \mathrm{a}=\sum \mathrm{1} /(i-1)
$$

where $\mathrm{K}$ is the number of SNPs identified in an alignment length, $\mathrm{n}$ is alleles and $\mathrm{L}$ is the total length of sequence (bp).

\section{F.Data analyses}

Data from ammonium, orthophosphate and IAA concentrations in media were analysed in completely randomized design with three replicates. Yield component and grain yield together with $\mathrm{pH}$ and soil characteristics were analysed with five replications. Duncan test at $\mathrm{P}=0.01$ or $\mathrm{P}=0.05$ were used to differentiate between statistically different means using SPSS version 16.

\section{RESULSTS AND DISCUSSION}

\section{A.Plant sample and Isolation endophytic bacteria in soybean plant samples}

From colonies were plated and develop on TSA and PDA media after incubation at $30^{\circ} \mathrm{C}$; we wished to isolate non-rhizobia from within soybean plant samples. Total of 86 endophytic bacterial isolates (consisted of 41 isolates from Thot Not, 19 isolates from O Mon, 6 from Co Do, 11 from Vinh Thanh and 9 from Thoi Lai); 32 isolates isolated on PDA medium, 42 isolates on TSA medium and 12 isolates on G6 medium. The endophytic bacteria developed in the pellicles of semi solid (in two kinds of medium) after $36 \mathrm{~h}$ incubation in semi-solid (Figure 2) as the previous results of Thu Ha et al. [25], Diep et al [26].

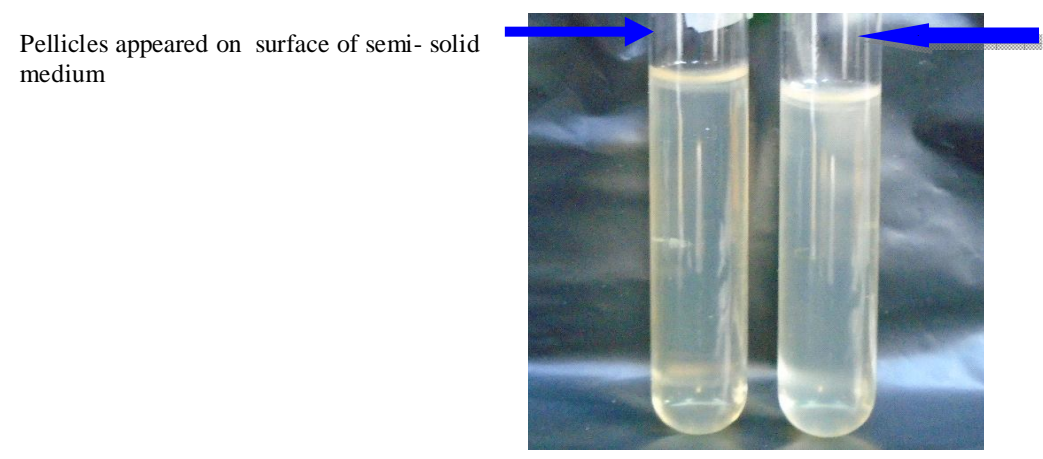

Figure - 2 Endophytic bacteria made pellicles in semi-solid (TSA and PDA media) after $36 \mathrm{~h}$ incubation at $30^{\circ} \mathrm{C}$ 
Almost their colonies have round-shaped; milky, white clear (on PDA's medium and TSA medium); entire or loabate margin (Figure 3); diameter size of these colonies varied from 0.2 to $3.0 \mathrm{~mm}$ and all of them are Grampositve or Gram-negative by Gram stain.

Figure - 3 Characteristics of colonies of bacterial isolates after grown on two kinds of medium

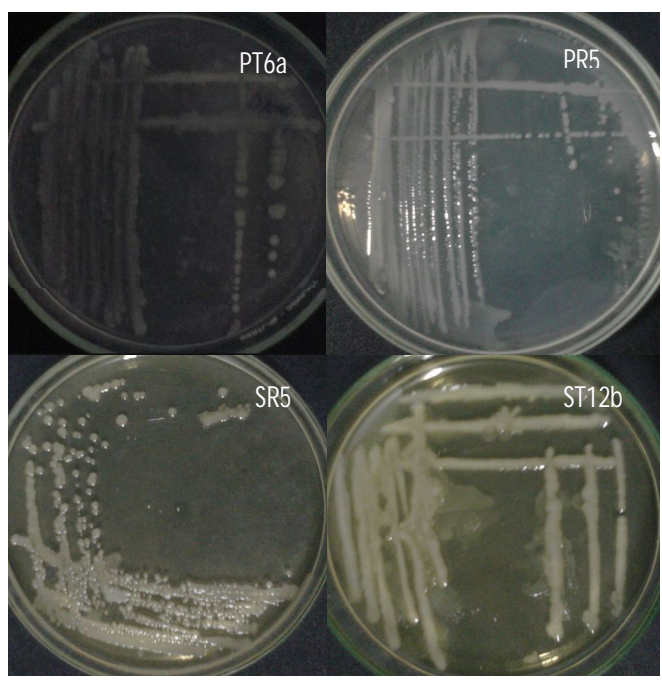

\section{B. Characterization of endophytic bacteria for plant growth promoting attributes}

The result from Table 1 showed that there were some isolates having the highest ammonium concentration as isolates TPR6a, TPR7a, TPR7b, TPT7, TPT8, CPT1A and CPT2A on PDA medium, TSR5, TSR6b, TSR6b, TSR12, TST6c, TST10b, TSR2B, VSR2A on TSA medium (Table 2) and TGN1, OGN6 on G6 medium (Table 3) however there were a lot of isolates having the high ability of phosphate solubilization such as isolates CPR1A, TPN1A, CPT1A on PDA medium (Table 4), CSR2B, VST1A1 on PDA medium (Table 5) and the isolate VGN2 on G6 medium (Table 6).

Table - 1 Ammonium concentration (mg/L) of some good bacterial isolates on PDA medium from 5 sites of Can Tho city

\begin{tabular}{|c|c|c|c|c|c|c|c|c|}
\hline No & Bacterial name & Day 2 & Day 4 & & ay 6 & & ay 8 & Site \\
\hline 01 & Control & $0.000 \quad \mathrm{j}^{\mathrm{j}}$ & 0.000 & 0.004 & $\mathrm{j}$ & 0.001 & $\mathrm{j}$ & \\
\hline 03 & TPR6a & $3.111 \mathrm{c}$ & $0.131 \quad \mathrm{~h}$ & 0.005 & $\mathrm{j}$ & 0.001 & $\mathrm{j}$ & Thot Not \\
\hline 04 & TPR6b & $1.382 \mathrm{f}$ & 0.081 & 0.021 & $\mathrm{i}$ & 0.031 & $\mathrm{i}$ & Thot Not \\
\hline 05 & TPR7a & $6.001 \mathrm{a}$ & $4.012 \mathrm{~b}$ & 0.003 & $\mathrm{j}$ & 0.031 & $\mathrm{i}$ & Thot Not \\
\hline 06 & TPR7b & $4.221 \mathrm{~b}$ & $0.051 \quad \mathrm{i}$ & 0.002 & $\mathrm{j}$ & 0.051 & $\mathrm{i}$ & Thot Not \\
\hline 07 & TPR9b & $3.372 \mathrm{c}$ & $2.981 \mathrm{c}$ & 0.001 & $\mathrm{j}$ & 0.141 & $\mathrm{~h}$ & Thot Not \\
\hline 08 & TPR10a & $2.201 \mathrm{~d}$ & $0.181 \mathrm{~g}$ & 0.002 & $\mathrm{j}$ & 0.131 & $\mathrm{~h}$ & Thot Not \\
\hline 09 & ТРТ6а & $4.021 \mathrm{~b}$ & $0.722 \mathrm{f}$ & 0.004 & $\mathrm{j}$ & 0.121 & $\mathrm{~h}$ & Thot Not \\
\hline 10 & TPT7 & $4.001 \mathrm{~b}$ & $3.861 \mathrm{bc}$ & 0.003 & $\mathrm{j}$ & 0.052 & $\mathrm{i}$ & Thot Not \\
\hline 11 & TPT8 & $4.152 \mathrm{~b}$ & 0.091 & 0.001 & $\mathrm{j}$ & 0.051 & $\mathrm{i}$ & Thot Not \\
\hline 12 & TPТ10 & $3.151 \mathrm{c}$ & 0.092 & 0.001 & $\mathrm{j}$ & 0.031 & $\mathrm{i}$ & Thot Not \\
\hline 14 & CPR1A & $0.493 \mathrm{fg}$ & 0.008 & 0.434 & $\mathrm{fg}$ & 0.966 & $\mathrm{f}$ & Co Do \\
\hline 15 & CPR2A & $0.775 \quad \mathrm{f}$ & $0.707 \quad \mathrm{f}$ & 0.345 & $\mathrm{~g}$ & 1.049 & ef & Co Do \\
\hline 16 & VPR2B & $1.066 \mathrm{e}$ & 0.008 & 0.209 & $\mathrm{~h}$ & 1.254 & ef & Vinh Thanh \\
\hline 17 & VPR1B & $1.266 \mathrm{e}$ & $3.973 \mathrm{~b}$ & 0.936 & ef & 1.391 & e & Vinh Thanh \\
\hline
\end{tabular}




\begin{tabular}{|l|l|l|l|l|l|l|}
\hline 18 & LPN1 A & $1.257 \mathrm{e}$ & 0.973 ef & $2.732 \mathrm{~d}$ & $1.687 \mathrm{e}$ & Thoi Lai \\
\hline 19 & LPN2A & $1.246 \mathrm{e}$ & $3.209 \mathrm{c}$ & $0.156 \mathrm{~h}$ & $0.756 \mathrm{f}$ & Thoi Lai \\
\hline 20 & CPT1A & $1.377 \mathrm{e}$ & $2.397 \mathrm{~d}$ & $0.529 \mathrm{f}$ & 1.094 ef & Co Do \\
\hline 21 & CPT2A & $1.447 \mathrm{e}$ & $6.455 \mathrm{a}$ & $0.004 \mathrm{j}$ & $0.481 \mathrm{fg}$ & Co Do \\
\hline \multicolumn{7}{|l|}{ C.V $=6.42 \%$} \\
\hline
\end{tabular}

Means within a column followed by the same letter/s are not significantly different at $\mathrm{p}<0.01$

Table - 2 Ammonium concentration ( $\mathrm{mg} / \mathrm{L}$ ) of some good bacterial isolates on TSA medium from 5 sites of Can Tho city

\begin{tabular}{|c|c|c|c|c|c|c|c|}
\hline No & Bacterial name & Day 2 & Day 4 & & Day 6 & Day 8 & Site \\
\hline 01 & Control & $0.000 \quad \mathrm{n}$ & $0.000 \quad \mathrm{n}$ & 0.000 & $\mathrm{n}$ & 0.000 & \\
\hline 02 & TSR5 & $6.341 \mathrm{~b}$ & $6.391 \mathrm{~b}$ & 2.091 & $\mathrm{f}$ & 0.321 & Thot Not \\
\hline 03 & TSR6a & $1.472 \mathrm{~g}$ & $0.121 \mathrm{~m}$ & 0.112 & $\mathrm{~m}$ & 0.102 & Thot Not \\
\hline 04 & TSR6b & $7.261 \mathrm{a}$ & $0.112 \mathrm{~m}$ & 0.102 & $\mathrm{~m}$ & 0.111 & Thot Not \\
\hline 05 & TSR10b & $3.721 \mathrm{e}$ & $0.082 \mathrm{~m}$ & 0.082 & $\mathrm{~m}$ & 0.031 & Thot Not \\
\hline 06 & TSR12 & $7.481 \mathrm{a}$ & $0.152 \mathrm{~m}$ & 0.041 & $\mathrm{~m}$ & 0.031 & Thot Not \\
\hline 07 & TST6c & $5.221 \mathrm{c}$ & $3.342 \mathrm{e}$ & 0.051 & $\mathrm{~m}$ & 0.082 & Thot Not \\
\hline 08 & TST7a & $2.302 \mathrm{f}$ & $0.131 \quad \mathrm{~m}$ & 0.103 & $\mathrm{~m}$ & 0.112 & Thot Not \\
\hline 09 & TST8a & $2.842 \mathrm{f}$ & $0.092 \mathrm{~m}$ & 0.121 & $\mathrm{~m}$ & 0.061 & Thot Not \\
\hline 10 & TST8b & $6.223 \mathrm{~b}$ & $0.052 \mathrm{~m}$ & 0.104 & $\mathrm{~m}$ & 0.061 & Thot Not \\
\hline 11 & TST10a & $4.031 \mathrm{~d}$ & $0.092 \mathrm{~m}$ & 0.041 & $\mathrm{~m}$ & 0.041 & Thot Not \\
\hline 12 & TST10b & $3.052 \mathrm{ef}$ & $4.041 \mathrm{~d}$ & 0.251 & 1 & 0.172 & Thot Not \\
\hline 13 & CSR2B & $1.427 \mathrm{~g}$ & $0.057 \quad \mathrm{~m}$ & 0.053 & $\mathrm{~m}$ & 0.860 & Co Do \\
\hline 14 & VST1A1 & $0.996 \quad$ hi & $2.371 \quad \mathrm{f}$ & 0.336 & $\mathrm{k}$ & $1.513 \mathrm{~g}$ & Vinh Thanh \\
\hline 15 & VST1A2 & $0.795 \quad \mathrm{i}$ & $1.204 \mathrm{gh}$ & 0.015 & $\mathrm{n}$ & $1.011 \mathrm{gh}$ & Vinh Thanh \\
\hline 16 & VSR1A & $1.058 \mathrm{gh}$ & $1.419 \mathrm{~g}$ & 0.379 & $\mathrm{k}$ & $1.091 \mathrm{gh}$ & Vinh Thanh \\
\hline 17 & VST1A & $1.114 \mathrm{gh}$ & $3.804 \mathrm{e}$ & 1.022 & $\mathrm{gh}$ & 0.528 & Vinh Thanh \\
\hline 18 & VST2A & $0.783 \quad \mathrm{i}$ & $6.009 \mathrm{~b}$ & 0.633 & $\mathrm{i}$ & 0.299 & Vinh Thanh \\
\hline 19 & LSR2B & 0.437 & $7.734 \mathrm{a}$ & 0.536 & $\mathrm{j}$ & $1.183 \mathrm{gh}$ & Thoi Lai \\
\hline 20 & VSR2A & $0.027 \quad n$ & $7.029 \mathrm{a}$ & 0.041 & $\mathrm{mn}$ & 0.401 & Vinh Thanh \\
\hline 21 & CST2B & $0.776 \quad j$ & $5.212 \mathrm{c}$ & 0.184 & $\mathrm{~m}$ & 0.516 & Co Do \\
\hline 22 & VST2B & $0.351 \quad \mathrm{k}$ & $1.363 \mathrm{~g}$ & 2.096 & $\mathrm{f}$ & 0.457 & Vinh Thanh \\
\hline 23 & LSR1A & $0.541 \quad \mathrm{j}$ & $6.482 \mathrm{~b}$ & 3.536 & $\mathrm{e}$ & 0.502 & Thoi Lai \\
\hline 24 & LSR2A & $1.867 \mathrm{~g}$ & $2.831 \mathrm{f}$ & 0.217 & $\mathrm{i}$ & 0.096 & Thoi Lai \\
\hline
\end{tabular}

Means within a column followed by the same letter/s are not significantly different at $\mathrm{p}<0.01$

Table - 3 Ammonium concentration (mg/L) of some good bacterial isolates on G6 medium from 4 sites of Can Tho city

\begin{tabular}{|c|c|c|c|c|c|c|c|c|c|}
\hline \multirow{2}{*}{$\begin{array}{l}\text { No } \\
01\end{array}$} & \multirow{2}{*}{$\begin{array}{l}\text { Bacterial name } \\
\text { Control }\end{array}$} & \multicolumn{2}{|c|}{ Day 2} & Day 4 & \multicolumn{2}{|c|}{ Day 6} & \multicolumn{2}{|c|}{ Day 8} & \multirow[t]{2}{*}{ Site } \\
\hline & & 0.000 & $\mathrm{n}$ & 0.000 & 0.000 & $\mathrm{n}$ & 0.000 & $\mathrm{n}$ & \\
\hline 02 & TGN1 & $4.031 \mathrm{~b}$ & & $3.441 \mathrm{c}$ & 0.001 & $\mathrm{n}$ & 0.041 & $\mathrm{~m}$ & Thot Not \\
\hline 03 & OGN2 & $3.291 \mathrm{c}$ & & $3.291 \mathrm{c}$ & 0.071 & $\mathrm{~m}$ & 0.051 & $\mathrm{~m}$ & O Mon \\
\hline 04 & OGN3 & 0.281 & 1 & $0.732 \mathrm{~d}$ & 0.033 & $\mathrm{n}$ & 0.002 & $\mathrm{n}$ & O Mon \\
\hline 05 & OGN4 & 0.081 & $\mathrm{~m}$ & 0.521 ef & 0.003 & $\mathrm{n}$ & 0.022 & $\mathrm{n}$ & O Mon \\
\hline 06 & OGN5 & 0.102 & 1 & $0.371 \quad \mathrm{fg}$ & 0.011 & $\mathrm{n}$ & 0.001 & $\mathrm{n}$ & O Mon \\
\hline 07 & OGN6 & $6.961 \mathrm{a}$ & & $6.961 \mathrm{a}$ & 0.003 & $\mathrm{n}$ & 0.003 & $\mathrm{n}$ & O Mon \\
\hline 08 & OGN7 & 0.191 & 1 & $0.381 \quad \mathrm{fg}$ & 0.011 & $\mathrm{n}$ & 0.012 & $\mathrm{n}$ & O Mon \\
\hline 09 & OGN8 & 0.121 & 1 & $0.341 \quad \mathrm{~g}$ & 0.003 & $\mathrm{n}$ & 0.012 & $\mathrm{n}$ & O Mon \\
\hline 10 & TGN9 & $0.921 \mathrm{~d}$ & & $0.622 \mathrm{~d}$ & 0.002 & $\mathrm{n}$ & 0.003 & $\mathrm{n}$ & Thot Not \\
\hline 11 & LGN1 & 0.025 & $\mathrm{~m}$ & $3.019 \mathrm{c}$ & 0.044 & $\mathrm{~m}$ & 0.255 & $\mathrm{~g}$ & Thoi Lai \\
\hline 12 & LGN2 & 0.032 & $\mathrm{~m}$ & $3.058 \mathrm{c}$ & 0.041 & $\mathrm{~m}$ & 0.367 & $\mathrm{fg}$ & Thoi Lai \\
\hline 13 & VGN2 & 0.156 & 1 & $0.424 \quad f$ & $0.949 \mathrm{~d}$ & & 0.457 & $\mathrm{f}$ & Vinh Thanh \\
\hline \multicolumn{10}{|c|}{$\mathrm{CV}=5.96 \%$} \\
\hline
\end{tabular}


Means within a column followed by the same letter/s are not significantly different at $\mathrm{p}<0.01$

Table - 4 Phosphate concentration (mg/L) of some bacterial isolates on PDA medium from 5 sites of Can Tho city

\begin{tabular}{|c|c|c|c|c|c|c|}
\hline No & Bacterial name & Day 5 & Day 10 & Day 15 & Day 20 & Site \\
\hline 01 & Control & $00.00 \quad \mathrm{f}$ & $00.00 \quad \mathrm{f}$ & $00.00 \quad \mathrm{f}$ & $00.00 \quad \mathrm{f}$ & \\
\hline 02 & TPR7b & 19.50 ef & $48.61 \mathrm{de}$ & $42.93 \mathrm{de}$ & $54.20 \mathrm{de}$ & Thot Not \\
\hline 03 & TPR9c & $30.79 \mathrm{e}$ & $45.79 \mathrm{de}$ & $45.97 \mathrm{de}$ & $73.64 \mathrm{~d}$ & Thot Not \\
\hline 04 & TPT9 & $34.11 \mathrm{e}$ & $40.95 \mathrm{de}$ & $41.86 \mathrm{de}$ & $52.26 \mathrm{~d}$ & Thot Not \\
\hline 05 & TPT11 & $32.63 \mathrm{e}$ & $64.86 \mathrm{~d}$ & $63.56 \mathrm{~d}$ & $93.22 \mathrm{~d}$ & Thot Not \\
\hline 06 & TPN9 & $00.82 \mathrm{f}$ & $152.84 \mathrm{c}$ & 18.37 & 11.64 & Thot Not \\
\hline 07 & CPR1A & $259.94 \mathrm{~b}$ & $467.48 \mathrm{a}$ & $235.28 \mathrm{~b}$ & $266.67 \mathrm{~b}$ & Co Do \\
\hline 08 & LPN1A & $245.61 \mathrm{~b}$ & $212.34 \mathrm{~b}$ & $416.94 \mathrm{a}$ & 85.51 & Thoi Lai \\
\hline 09 & CPT1A & $320.80 \mathrm{~b}$ & $72.84 \mathrm{~d}$ & $322.57 \mathrm{~b}$ & 00.56 & Co Do \\
\hline
\end{tabular}

Means within a column followed by the same letter/s are not significantly different at $\mathrm{p}<0.01$

Table - 5 Phosphate concentration $(\mathrm{mg} / \mathrm{L})$ of some good bacterial isolates on TSA medium from 5 sites of Can Tho city

\begin{tabular}{|c|c|c|c|c|c|c|}
\hline No & Bacterial name & Day 5 & Day 10 & Day 15 & Day 20 & Site \\
\hline 01 & Control & $00.00 \quad h$ & $00.00 \quad h$ & 00.00 & 00.00 & \\
\hline 02 & TSR6b & $49.79 \quad \mathrm{~g}$ & $49.79 \mathrm{~g}$ & $41.47 \quad \mathrm{f}$ & $37.61 \quad \mathrm{~g}$ & Thot Not \\
\hline 03 & TSR10c & $01.23 \quad \mathrm{~h}$ & $47.73 \quad \mathrm{~g}$ & $54.78 \quad \mathrm{~g}$ & $84.30 \quad \mathrm{e}$ & Thot Not \\
\hline 04 & TST5 & $02.38 \quad \mathrm{~h}$ & $53.83 \quad \mathrm{~g}$ & $64.20 \quad \mathrm{fg}$ & $93.58 \mathrm{e}$ & Thot Not \\
\hline 05 & TST7a & $40.58 \quad \mathrm{~g}$ & $54.04 \quad \mathrm{~g}$ & $41.16 \quad \mathrm{~g}$ & 50.93 & Thot Not \\
\hline 06 & TST9 & $44.30 \mathrm{~g}$ & $49.64 \mathrm{~g}$ & $42.61 \quad \mathrm{~g}$ & 53.97 & Thot Not \\
\hline 07 & TST10c & $31.25 \mathrm{~g}$ & $44.45 \quad \mathrm{~g}$ & $44.60 \quad \mathrm{~g}$ & 67.91 & Thot Not \\
\hline 08 & OST11a & 67.91 & $51.87 \mathrm{~g}$ & $35.14 \quad \mathrm{~g}$ & 66.06 & O Mon \\
\hline 09 & OST11b & $43.35 \mathrm{~g}$ & $50.71 \mathrm{~g}$ & $40.40 \quad \mathrm{~g}$ & 51.43 & O Mon \\
\hline 10 & OST12a & $40.03 \mathrm{~g}$ & $44.30 \mathrm{~g}$ & $37.29 \quad \mathrm{~g}$ & 52.75 & O Mon \\
\hline 11 & CSR2B & $244.44 \mathrm{~d}$ & $135.18 \mathrm{e}$ & $310.27 \mathrm{~cd}$ & 55.07 & Co Do \\
\hline 12 & VST1A1 & $205.29 \mathrm{de}$ & 56.17 & $755.83 \mathrm{a}$ & 34.49 & Vinh Thanh \\
\hline 13 & VST1A & $111.37 \quad \mathrm{e}$ & $64.24 \quad \mathrm{~g}$ & $339.44 \mathrm{~cd}$ & 00.55 & Vinh Thanh \\
\hline 14 & VST2A & $188.76 \mathrm{de}$ & $168.93 \mathrm{e}$ & $434.72 \mathrm{c}$ & 00.56 & Vinh Thanh \\
\hline 15 & CST2B & $199.48 \mathrm{~d}$ & $84.85 \quad \mathrm{f}$ & $276.94 \quad \mathrm{~d}$ & $152.17 \mathrm{de}$ & Co Do \\
\hline 16 & VST2B & $204.39 \mathrm{~d}$ & $01.60 \quad \mathrm{~h}$ & $571.38 \mathrm{~b}$ & 65.25 & Vinh Thanh \\
\hline 17 & LSR2A & $257.11 \mathrm{~d}$ & $81.27 \quad \mathrm{f}$ & $751.11 \mathrm{a}$ & $167.25 \mathrm{de}$ & Thoi Lai \\
\hline 18 & LSR1A & $174.55 \mathrm{de}$ & $78.39 \quad \mathrm{f}$ & $486.66 \mathrm{bc}$ & 00.85 & Thoi Lai \\
\hline \multicolumn{7}{|c|}{$\mathrm{CV}=5.2 \%$} \\
\hline
\end{tabular}

Means within a column followed by the same letter/s are not significantly different at $\mathrm{p}<0.01$

Table - 6 Phosphate concentration (mg/L) of some good bacterial isolates on G6 medium from 4 sites of Can Tho city

\begin{tabular}{|c|c|c|c|c|c|c|}
\hline No & Bacterial name & Day 5 & Day 10 & Day 15 & Day 20 & Site \\
\hline 01 & Control & $00.00 \quad \mathrm{~g}$ & 00.00 & 00.00 & $00.00 \quad \mathrm{~g}$ & \\
\hline 02 & TGN1 & $36.64 \mathrm{f}$ & $56.31 \mathrm{f}$ & $46.79 \quad \mathrm{f}$ & $70.19 \mathrm{e}$ & Thot Not \\
\hline 03 & OGN6 & $32.81 \mathrm{f}$ & $57.56 \mathrm{f}$ & $56.83 \mathrm{f}$ & $115.75 \mathrm{~d}$ & O Mon \\
\hline 04 & LGN1 & $96.89 \mathrm{e}$ & $124.69 \mathrm{~d}$ & $471.71 \mathrm{a}$ & 00.36 & Thoi Lai \\
\hline 05 & VGN2 & $120.28 \mathrm{~d}$ & 01.29 & $282.22 \mathrm{~b}$ & $178.55 \mathrm{c}$ & Vinh Thanh \\
\hline
\end{tabular}

Means within a column followed by the same letter/s are not significantly different at $\mathrm{p}<0.01$ 
Especially, there were a lot of isolates having high IAA biosynthesis ability in condition without tryptophan such as TPR6b, TPR7b, TPR9c, TPR10c, TPT6a, TPT8 (on PDA medium), TST9, TST10c, TST11a, TST8c (TSA medium) and OGN6, OGN7 (G6 medium) (Table 7).

Table - 7 IAA concentration ( $\mu \mathrm{g} / \mathrm{L})$ of some good bacterial isolates on three kinds of medium from 5 sites of Can Tho city

\begin{tabular}{|c|c|c|c|c|c|c|}
\hline No & Bacterial name & Day 2 & Day 4 & Day 6 & Day 8 & medium \\
\hline 01 & Control & 0.00 & 0.00 & 0.00 & 0.00 & \\
\hline 02 & TPR6b & $15.40 \mathrm{c}$ & $8.91 \mathrm{de}$ & $12.63 \mathrm{~cd}$ & 6.45 ef & PDA \\
\hline 03 & TPR7b & 8.07 e & $7.64 \mathrm{e}$ & 2.87 gh & $28.88 \mathrm{a}$ & PDA \\
\hline 04 & TPR9c & $15.73 \mathrm{c}$ & $9.72 \mathrm{~d}$ & $15.34 \mathrm{c}$ & $27.46 \mathrm{a}$ & PDA \\
\hline 05 & TPR10a & $16.43 \mathrm{c}$ & $16.10 \mathrm{c}$ & 5.96 ef & $16.81 \mathrm{c}$ & PDA \\
\hline 06 & TPT6a & $12.69 \mathrm{~cd}$ & 6.26 ef & 5.14 f & $8.32 \mathrm{e}$ & PDA \\
\hline 07 & TPT7 & $11.08 \mathrm{~d}$ & 4.01 & $7.22 \mathrm{e}$ & 3.55 & PDA \\
\hline 08 & TPT8 & $15.60 \mathrm{c}$ & $12.25 \mathrm{~cd}$ & $9.15 \mathrm{~d}$ & $18.21 \mathrm{~b}$ & PDA \\
\hline 09 & CPR1A & $5.53 \mathrm{f}$ & 0.47 & 0.03 & 0.56 & PDA \\
\hline 10 & CPR2A & 6.15 ef & 0.60 & 0.07 & 0.07 & PDA \\
\hline 11 & VPR2B & $3.85 \mathrm{~g}$ & 0.05 & 0.04 & 0.05 & PDA \\
\hline 12 & VPR1B & $5.66 \mathrm{f}$ & 0.84 & 0.30 & 0.56 & PDA \\
\hline 13 & LPN1A & $5.32 \mathrm{f}$ & 0.50 & 0.40 & 0.66 & PDA \\
\hline 14 & VPR2A & 6.51 ef & 0.56 & $0.98 \quad \mathrm{~h}$ & 0.30 & PDA \\
\hline 15 & CPT1A & $2.66 \quad \mathrm{~h}$ & 0.08 & 0.07 & 0.06 & PDA \\
\hline 16 & CSR2B & $1.45 \quad \mathrm{~h}$ & 0.11 & $1.83 \mathrm{~h}$ & 0.45 & TSA \\
\hline 17 & LSR2A & $1.77 \quad h$ & 0.04 & 0.05 & 0.49 & TSA \\
\hline 18 & TST9 & $12.42 \mathrm{~cd}$ & $3.73 \quad \mathrm{~g}$ & $12.39 \mathrm{~cd}$ & $5.11 \mathrm{f}$ & TSA \\
\hline 19 & TST10c & $12.56 \mathrm{~cd}$ & 6.25 ef & $10.50 \mathrm{~d}$ & $7.10 \mathrm{e}$ & TSA \\
\hline 20 & TST11a & $14.20 \mathrm{c}$ & 8.19 e & $8.11 \mathrm{~d}$ & $5.98 \mathrm{f}$ & TSA \\
\hline 21 & TSN8c & $11.79 \mathrm{~d}$ & 4.17 & $10.08 \mathrm{~d}$ & 8.46 e & TSA \\
\hline 22 & TSR9c & $11.45 \mathrm{~d}$ & 4.16 & 5.52 & 1.07 & TSA \\
\hline 23 & TGN1 & $8.74 \mathrm{e}$ & 0.16 & $8.96 \mathrm{~d}$ & $5.43 \mathrm{f}$ & G6 \\
\hline 24 & OGN6 & $13.82 \mathrm{~cd}$ & $2.26 \quad \mathrm{~h}$ & $4.00 \quad \mathrm{~g}$ & 1.98 & G6 \\
\hline 25 & OGN7 & $2.93 \mathrm{gh}$ & $1.11 \mathrm{~h}$ & $2.67 \quad \mathrm{~h}$ & 21.16 b & G6 \\
\hline 26 & VGN2 & $1.47 \quad \mathrm{~h}$ & 0.04 & 0.03 & 0.93 & G6 \\
\hline
\end{tabular}

Means within a column followed by the same letter/s are not significantly different at $\mathrm{p}<0.01$

33/86 isolates $(38.37 \%)$ produced siderophores after 2 days incubated on CAS medium (Figure 4) with 9 isolates on PDA medium, 17 isolates on TSA medium and 7 isolates on G6 medium.

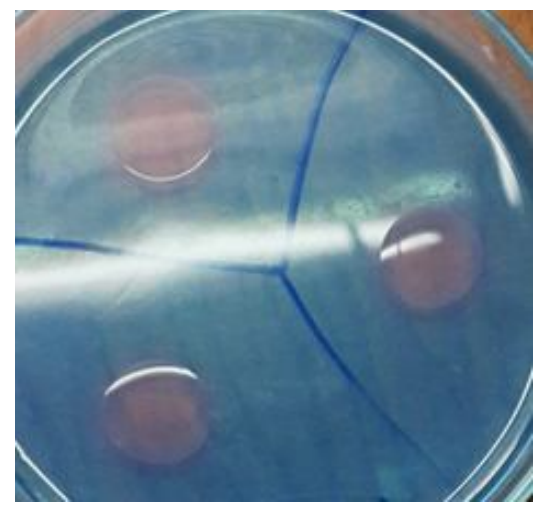

Volume 8 Issue 3 June 2017

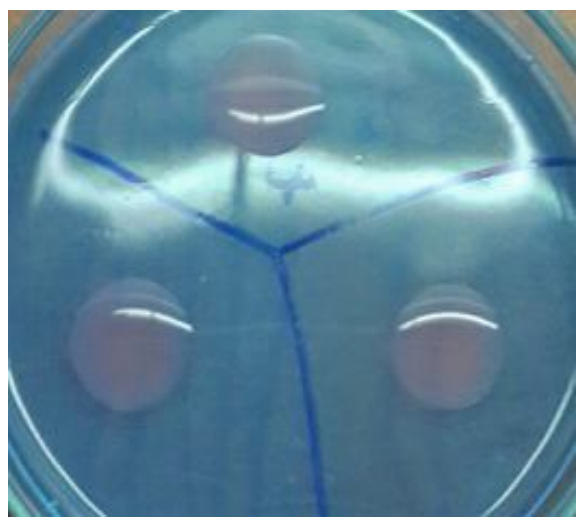


Figure 4. Bacterial isolates made a range of halo round well contaning bacterial liquid on CAS agar after $48 \mathrm{~h}$ incubation

Based on the characteristics as high nitrogen fixation, phosphate solublization, IAA and siderophores, 26 good isolates were chosen to identify with universal primers $27 \mathrm{~F}$ and $1492 \mathrm{R}$ and sequencing as TSR10c, TSR9c, TGN1, OGN6, TST5, TPR6a, TST6c, TST10c, TPN9, TSR6b, TPN10b, TSR12, TST11a, TST12a, TST10b, CPT1a, TSR1a, VST1a, VST2b, and VST1a.

The fragments of $900 \mathrm{bp} 16 \mathrm{~S}$ rRNA were obtained from PCR with p515FPL and p13B primers and sequencing. Homology searches of 16S rRNA gene sequence of selected strain in GenBank by BLAST revealved that they had similarity to sequences of Bacilli and Gammaproteobacteria (7/26 isolates and 19/26 isolates, respectively) (Figure 5) (Table 6).

Table - 6 Phylogenetic affiliation of isolates on the basis of 16S rRNA genes sequences by using BLAST programme in the GenBank database based on sequences similarity

\begin{tabular}{|c|c|c|}
\hline Taxonomic group and strain & Closest species relative & Similarity $(\%)$ \\
\hline \multicolumn{3}{|l|}{ Bacilli } \\
\hline \multirow[t]{2}{*}{ TST10b } & Bacillus subtilis strain GBPI25 (KF862010) & 99 \\
\hline & Bacillus methylotrophicus strain GBPI_CDB76 (KT887215) & 99 \\
\hline \multirow[t]{2}{*}{ OST11a } & Bacillus flexus strain IK-MB14-518F (FJ906742) & 99 \\
\hline & Bacillus sp. IK-MB10-518F (FJ906738) & 99 \\
\hline \multirow[t]{2}{*}{ OSR12 } & Bacillus sp. IK-MB10-518F (FJ906738) & 99 \\
\hline & Bacillus megaterium strain p56_D01 (JQ835316) & 99 \\
\hline \multirow[t]{2}{*}{ TST10c } & Bacillus subtilis, strain CEES, isolate CEES\#12 (LN827667) & 99 \\
\hline & Bacillus amyloliquefaciens strain TCCB001 (KC755040) & 99 \\
\hline \multirow[t]{2}{*}{ VGN2 } & Bacillus amyloliquefaciens strain 3EC2C2 (EU304922) & 99 \\
\hline & Bacillus licheniformis strain 3EC4A14 (EU304939) & 99 \\
\hline \multirow[t]{2}{*}{ VST2B } & Staphylococcus sp. PR23304 (KX881396) & 99 \\
\hline & Staphylococcus cohnii strain CMU-BE03 (KX235336) & 99 \\
\hline \multirow[t]{2}{*}{ CST2B } & Staphylococcus xylosus strain 2B (KY992565) & 99 \\
\hline & Staphylococcus saprophyticus strain TA1 (KY992564) & 99 \\
\hline \multicolumn{3}{|l|}{ Gammaproteobacteria } \\
\hline \multirow[t]{2}{*}{ TST6c } & Acinetobacter calcoaceticus strain ZLynn1000-19 (KY316498) & 100 \\
\hline & Acinetobacter sp. TH216 (KT826396) & 100 \\
\hline \multirow[t]{2}{*}{ TST5 } & Acinetobacter soli strain MBR4 (JX966422) & 99 \\
\hline & Acinetobacter sp. strain UPMCB-A0020 (KY784622) & 99 \\
\hline \multirow[t]{2}{*}{ TPR6a } & Acinetobacter calcoaceticus strain ZLynn1000 (KY316498) & 99 \\
\hline & Acinetobacter sp. strain ZLynn500-6 (KY316494) & 99 \\
\hline \multirow[t]{2}{*}{ TSR9c } & Acinetobacter radioresistens strain D45 (KU922212) & 99 \\
\hline & Bacillus subtilis strain RG3 (KY088048) & 99 \\
\hline \multirow[t]{2}{*}{ TSR10c } & Acinetobacter calcoaceticus strain JO-1 (KF374680) & 99 \\
\hline & Acinetobacter sp. VITRSA1 (KF179101) & 99 \\
\hline \multirow[t]{2}{*}{ TGN1 } & Acinetobacter sp. strain ZLynn1000-14 (KY316497) & 99 \\
\hline & Acinetobacter pittii strain W26 (KY922994) & 99 \\
\hline \multirow[t]{2}{*}{ CPT1A } & Acinetobacter soli strain MBR4 (JX966422) & 99 \\
\hline & Acinetobacter sp. strain UPMCB-A0020 (KY784622) & 99 \\
\hline \multirow[t]{2}{*}{ TSR1A } & Enterobacter cloacae strain RCB732 (KT260944) & 99 \\
\hline & Enterobacter ludwigii strain B2 (KT153616) & 99 \\
\hline \multirow[t]{2}{*}{ TSR6b } & Enterobacter cloacae strain RCB730 (KT260942) & 99 \\
\hline & Enterobacter xiangfangensis strain B1 (MF083087) & 99 \\
\hline \multirow[t]{2}{*}{ OST12a } & Enterobacter cloacae strain EPS-14 (KY848821) & 99 \\
\hline & Enterobacter sp. strain W2-10 (KY496302) & 99 \\
\hline TPN10b & Enterobacter sp. JCM 28267 (LC133614) & 99 \\
\hline
\end{tabular}




\begin{tabular}{|l|c|c|}
\hline & Enterobacter ludwigii strain SDWH10 (KX640114) & 99 \\
\hline VST1A & Enterobacter cloacae strain strain 1FTK7 (KC335297) & 99 \\
\hline & Enterobacter hormaechei strain p62_E04 (JQ829397) & 99 \\
\hline VST1A1 & Enterobacter cloacae strain ECNIH5 (KY207545) & 99 \\
\hline & Enterobacter hormaechei strain SBANHCA2 (KY285185) & 99 \\
\hline CPR1A & Enterobacter cloacae strain S12 (KY595448) & 99 \\
\hline & Enterobacter sp. strain BAB-6019 (KY672863) & 99 \\
\hline TSR1A & Enterobacter cloacae strain RCB732 (KT260944) & 99 \\
\hline & Enterobacter xiangfangensis strain RPK35 (KX980457) & 99 \\
\hline TPN1A & Enterobacter cloacae strain KMB42 (KY458520) & 99 \\
\hline & Enterobacter asburiae strain 1897PAA001_E1 (KX885508) & 99 \\
\hline TPN2A & Enterobacter sp. strain P26 (KY084467) & 99 \\
\hline & Enterobacter cloacae strain KMB42 (KY458520) & 99 \\
\hline TPN9 & Klebsiella pneumonia strain NF82 (KP772067) & 99 \\
\hline & Klebsiella sp. Z13 (KF835726) & 100 \\
\hline OGN6 & Proteus mirabilis strain FCX7 (KU942502) & 100 \\
\hline
\end{tabular}

A neighbor-joining phylogenetic tree in these isolates showing the two clusters: A and B. Cluster A divided into two cluster A1 and A2. Derived from cluster A1, small cluster A11 had seven isolates with six isolates were Gammaproteobacteria among which three isolates having close relationship (Acinetobacter radioresisters TSR9c, Acinetobacter calcoaceticus TPR6a and A. calcoaceticus TST6c related closely with two other strains (Enetrobacter sp. TPN10b and Proteus mirabilis OGN6) while these five strains also had a relationship with Acinetobacter sp. TST5 and Bacillus sp. OST11a. Cluster A12 had three strains with two strains Enterobacter cloacae OST12a and E. cloacae TSR6b having close relationship, both related with strain Staphylococcus sp. VST2B. In addition, cluster A2 also had three strains as Enterobacter cloacae CPR1A, Enterobacter cloacae TSR2A and Enterobacter cloacae TPN2a having a close relationship and all of them related with strain Bacillus tequilensis TST10b.

Similarly, cluster B composed of two clusters: B1 and B2. Small cluster B1 with three strains such as Enterobacter cloacae VST1A, Enterobacter cloacae TPN1A and Bacillus sp. OSR12 had a close relationship. Cluster B2 with two cluster: cluster B21 comprised of two strains Bacillus amyloliquefasciens VGN2 and Staphylococcus xylosus CST2B, being Bacilli, whereas cluster B22 with two smaller clusters such as cluster B221 and cluster B222. Cluster B221 with three strains Enterobacter cloacae TSR1A, Enterobacter cloacae VST1A1 and Acinetobacter soli CPT1A had a close relationship. Especially, in cluster B222, two strains as Bacillus subtilis TST10c and Acinetobacter calcoaceticus TSR10c (TST came from soybean plant whilst TSR stemmed from soybean root) belonged to a cluster; whereas other strains Klebsiella pneumonise TPN9 and Acinetobacter sp. TGN1 arose from another cluster.

These results showed that the strains presented a very close relationship between two endophytic bacterial strains having a relationship in soybean plant but we are not sure that they were same species, same genus or same Gram-positive/Gram-negative. 


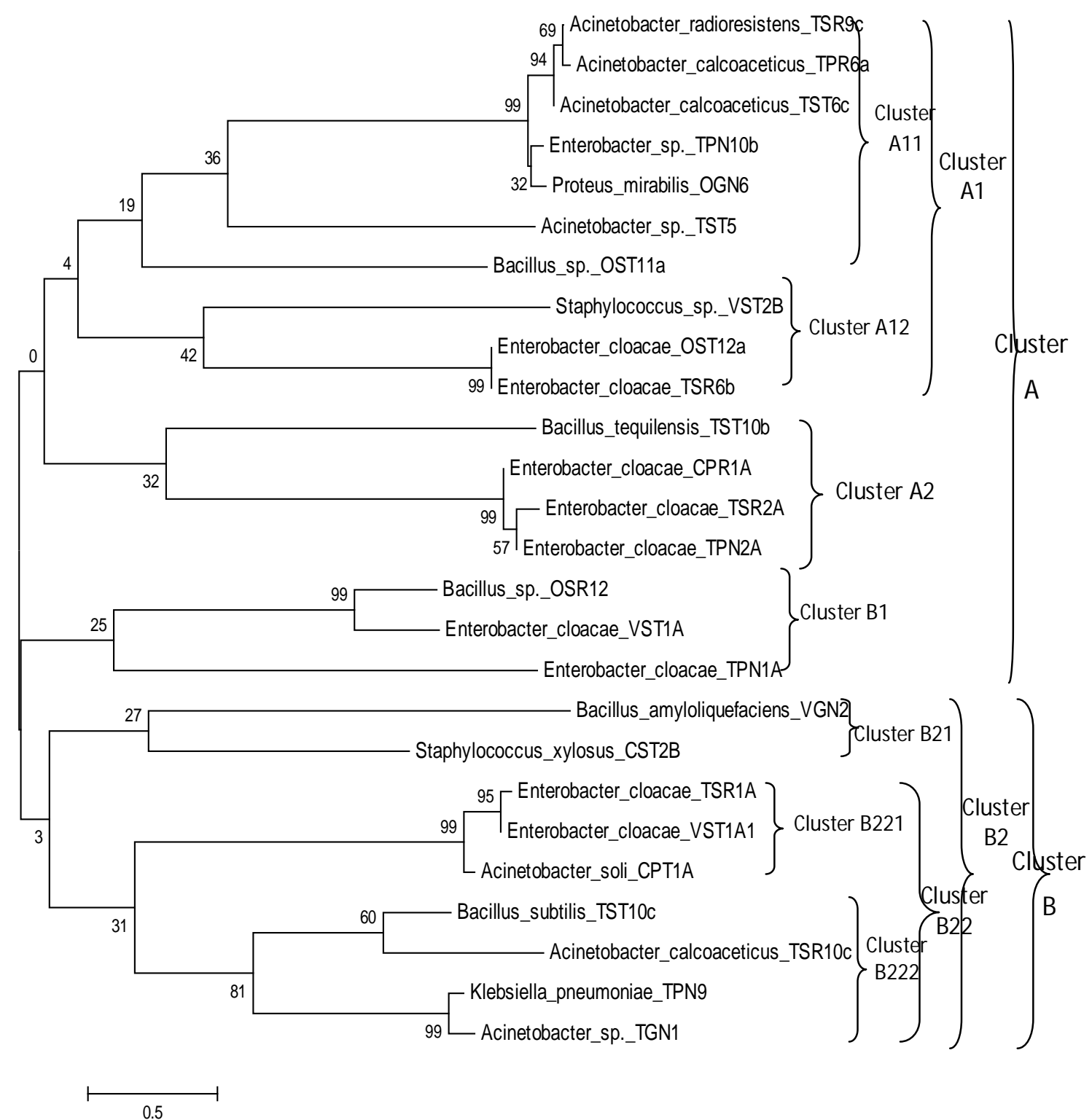

Figure 3. Phylogenetic tree showing the relative position of endophytic bacterial isolates by the neighbor-joining method of complete $16 \mathrm{~S}$ rRNA sequence (p515FPL primer)

Bootstrap values of 1000 replicates are shown at the nodes of the trees.

Among 26 strains, there were 21 strains having length nucleotide (over 600) and Theta values (per sequence) from S of SNP for DNA polymorphism were calculated for Each group, and Gammaproteobacteria group had the highest values as comparison with Bacilli (Table 7). Nucleotide polymorphism can be measured by many parameters, such as halotypes (genes) diversity, nucleotide diversity, (Pi), Theta ( $\square$ )(per group) etc .In this study, nucleotide diversity was estimated by Theta $(\square)$, the number of segregating sites [27], and its standard deviation 
$(\mathrm{S} \square)$. These parameters were estimated by DNA Sequence Polymorphism software version 4.0 [28]. Pi values explained nucleotide diversity of sequences for each gene, the highest values, more diversity among groups.

Table - 7 Genetic diversity of 21 strains

\begin{tabular}{lccc}
\hline & Nucleotie diversity & Theta (per site) from Eta & Theta (per site) from S ( $\square$ ) \\
\hline 21 strains & 0.69990 & $0.79357 \pm 0.092$ & $0.27795 \pm 0.0085$ \\
\hline $\begin{array}{l}\text { Primer p515FPL } \\
\text { Primer p13B }\end{array}$ & 5'-AGGCCAGCAGCCGCGTAA-3' & & \\
\hline
\end{tabular}

The endophytic bacteria have been studied and described as beneficial bacteria with Bacilli and Gammaproteobacteria presented on LGI medium and it occupied 27\% and 73\% respectively in the total of 26 strains according to our result (Figure 5)

Figure - 5 The proportion of group and they distributed in two clusters

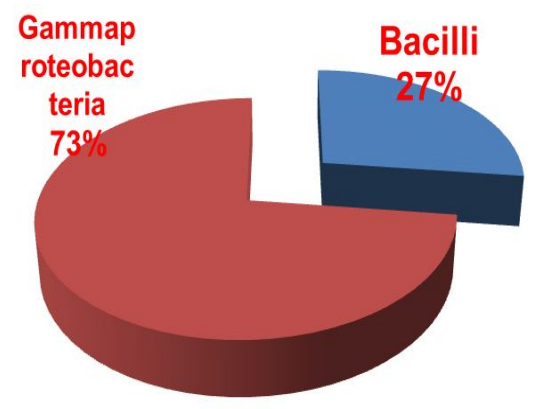

Soybean (Glycine max (L.) Merrill) is an Asiatic leguminous plant, occupying large acreages of land worldwide for its oil and protein [3]. Rhizobia are perhaps the best known beneficial plant-associated bacteria because of the importance of the nitrogen fixation that occurs during the Rhizobium-legume symbiosis [4]. Besides endophytic bacteria from legume plants have been reported; endophytic bacteria from roots and nodules of fieldpea and chickpea being grown in Northern India were isolated. A total of 75 endophytic bacteria roots and nodules of fieldpea [29] and 88 from roots and nodules of chickpea showed that 50\% in roots and $93.4 \%$ in nodules were Gram positive. Endophytic bacteria have been isolated from soybean [13] and especially Sturz et al. [11] reported the isolation of 15 non-rhizobial species from clover root nodules; Bai et al. [30] reported fourteen strains of putative endophytic bacteria, not including endosymbiotic Bradyrhizobium strains, were isolated from surface-sterilized soybean (Glycine max (L.) Merr.) root nodules; Hung et al. [4] found that endophytic population was highest in the nodules tissue with 31 nodule isolates, however, did not form nodules on soybean (cv. Pusa-22) and they suggested that 31 endophytic bacterial isolates. Hung et al, [4] reported that the isolation of Paenibacillus polymyxa HKA-15, a Gram-positive bacterium from root nodules of soybean and this strain showed that potent biocontrol activity towards soil borne fungal plant pathogens [31]. Our previous results [26] showed that over 50\% endophytic bacterial strains were idenfified that are bacilli among Paenibacillus lautus CJE17 which the best strain, it combined with the rhizobial strains as VNR71 or it combined with another strain (CJE10) supported grain yield.

In the our previous result, sixty-eight isolates were isolated from soybean nodules which were identified as endophytic bacterial isolates and 16 isolates having good plant growth promotion were chosen to analyse their relationship. These isolates were identified as Bacilli (more than 50\%), and Gammaproteobacteria with seven strains. Among them, there are two strains as Paenibacillus lautus CJE17 and Bacillus megaterium CJE10 supported yield component, grain yield and improved soil fertility of soybean cultivated on ferralsols [26]. However result of this study showed that Gammaproteobacteria occupied to $73 \%$ (nearly 3/4) and Bacilli was only $27 \%$, especially the endophytic bacterial strains in soybean phant or nodule are Bacilli and Gammaproteobacteria, the genus of bacilli and gammaproteobacteria distributed evenly whole soybean plant (nodule, root, stem) 
Based on biosafety and good characteristics as nitrogen fixation, phosphate solubilization, IAA synthesis, and siderophores production, Bacillus sp. OSR12, Bacillus subtilis TST10c, Acinetobacter sp. TGN1, Enterobacter cloacae TSR1A, Enterobacter cloacae CPR1A, were selected to evaluate their effects on pot-experiment and field trials; together with rhizobia into inoculant for soybean production in alluvial soil.

Compared to Gram-negative bacteria, Gram positive bacteria strains have the advantages as its ability to form endospores and produce different antibiotics. On the otherhand, Bacilli can survive for a long time in carrier in comparison with other bacteria in inoculant production commercially and especially endophytic bacterial Bacilli strains will be selected with characteristic of biological safety.

\section{IV.CONCLUSION}

Sixty-eight entophytic bacterial isolates were isolated from 70 soybean plant samples which collected at 5 districts: O Mon, Thot Not, Vinh Thanh, Co Do and Thoi Lai, Can Tho city, they developed on two kinds of medium (PDA and TSA) after 2 or 3 days incubation and they made the pellicles on semi-solid media. They were identified as endophytic bacterila isolates and 26 isolates having good plant growth promotion were chosen to analyse their relationship. These isolates were identified as Bacilli (27\%), and Gammaproteobacteria (73\%). Among them, there are five good strains (together with rhizobial strains), they will be suggested to produce as inoculant for soybean cultivation on aluvial soil in the future.

\section{ACKNOWLEDGEMENTS}

The authors thank the helpfulness of Microbiology BSc. Students and technicians in the Environment Microbiology Laboratory, Biotechnology R\&D Institute, Can Tho University, Vietnam; especially Associate Professor Dr. TRUONG TRONG NGON, Head of Molecular Biotechnology Department, Biotechnology R\&D Institute, Can Tho University, Vietnam for analysing molecular data, Ms. NGUYEN THI XUAN MY in the laboratory expriments and Ms. DAO THI MINH CHAU, Environment Microbiology Laboratory, Biotechnology R\&D Institute, Can Tho University, Vietnam for grammartical english.

\section{REFERENCE}

[01] Z. Fatima, M. Zia, and M.F. Chauhary, "Effect of Rhizobium and phosphorus on growth of soybean (Glycine max) and survival of Rhizobium and P solubilizing bacteria," Pak. J. Bul., vol. 38(2), pp: 459-464, 2006.

[02] M. Rana, P. Pathania and S.L. Khaswan, "Effect of biofertilizers and phosphorus on productivity and nutrient uptake of soybean (Glycine max L.)," Ann. Agric. Res. New Series vol. 34 (3), pp : 245-247, 2013.

[03] Fayzalla, E.A., El-Barougy, E; and El-Rayes, M.M., "Control of Soil-Borne Pathogenic Fungi of Soybean by Biofumigation with Mustard Seed Meal. J. Appl. Sci.; vol. 9,pp:2272-2279, 2009.

[04] P.Q. Hung, S.M. Kumar, V. Govindsamy, and A. Annapurna, "Isolation and characterization of endophytic bacteria from wild and cultivated soybean varieties" Biol. Fertil. Soils.; vol. 44,pp:155-162, 2007.

[05] S.S. Dudeja, and Giri, R, "Beneficial properties, colonization, establishment and molecular diversity of endohytic bacteria in legumes and non legumes," African Journal of Microbiology Research, vol. 8(15), pp:1562-1572, 2014.

[06] R.P. Ryan, K. Germaine, A. Franks, D. J. Ryan and D. N. Dowling, "Bacterial endophytes: recent developments and applications."FEMS Microbiol Lett. vol. 278(1), pp:1-9, 2008.

[07] D.Y. Kobayashi, and Palumbo, J.D, "Bacterial endophytes and their effects on plants and uses in agriculture", pp. 199-233, 2000. In: C.W. Bacon and J.F. White (ed.), Microbial Endophytes. Marcel Dekker, Inc, New York, N.Y.

[08] S. Hasegawa, A. Meguro, M. Shimizu, T. Nishimura, and H. Kunoch, "Endophytic actinomycetes and their interactions with host plants"' Actinomycetologica, vol. 20, pp:72-81, 2006.

[09] Z. Sapak, S. Meon, Z.A.M. Ahmad , "Effect of endophytic bacteria on growth and suppression of Ganoderma infection in oil palm," Int. J. Agri. Biol., vol.10, pp:127-32, 2008.

[10] S. Gagne, C. Richard, H. Roussem, and H. Antoun, "Xylem-residing bacteria in alfalfa roots" Can. J. Microbiol., vol.33,pp: 9961000, 1987.

[11] A.V. Sturz, and J. Nowak, "Endophytic communities of rhizobacteria and the strategies required to create yield enhancing associations with crops,” Appl. Soil. Ecol., vol.15, pp.183-190, 2000. 
[12] L. Elvira-Rucuenco, and J.W.L. van Vuurde, "Natural incidence of endophytic bacteria in pea cultivars under field condition. Can. J. Microbiol., vol. 46., pp: 1036-1041, 2000.

[13] N.W. Oehrle, D.B. Kart, R.J. Kremer, and D.W. Emerich, "Enhanced attachment of Bradyrhizobium japonicum to soybean through reduced root colonization of internally seedborne microorganisms" Can. J. Microbiol.; vol.46, pp.600-606, 2000.

[14] C.H. Collins, C.H., and P.M. Lyne, "Microbiological methods" 1984; Butterworths, London.

[15] J.M. Vincent, A manual for practical study of root nodule bacteria. Blackwell Scientific Publication, Oxford, pp:169-170, 1970.

[16] S.A. Gordon and R.P. Weber., "Colometric estimation of indolacetic acid,” Plant Physiol., vol 26, pp.192-195, 1951.

[17) H.M. Tam and C.N. Diep, "Isolation, Characterization and Identification of Endophytic Bacteria in Sugarcane (Saccharum spp. L.) Cultivated on Soils of the Dong Nai province, Southeast of Vietnam," American J. Life Science, vol.2, No.2 2014, pp. 361-368. doi: 10.11648/j.ails.2014206.16.

[18] B., Schwyn, and J.B. Neilands. "Universal chemical assay for the detection and determination of siderophores". Analytical Biochemistry, vol. 60(1), pp. 47-56, 1987.

[19] B. Neumann, A. Pospiech, and H.U. Schairrer, "Rapid isolation of genomic DNA from Gram-negative," Trends Gent., vol. 8, pp. 332-333, 1992.

[20] D.A. Relman, T.M. Schmidt, E.P. MacDermontt, and S. Falkow, "Identification of the uncultured bacillus of Whipple's disease," N. Engl. J. Med., vol. 327, pp.293-301, 1992.

[21] D.A. Relman, J.S. Loutit, T.M. Schmidt, S. Falkow, and L.S. Tompkin, "The agent of bacillary angiomatosis. An approach to the identification of uncultuted pathogens," N. Engl. J. Med., vol. 323, pp.1573-1580, 1990.

[22] D.K. Zinniel, , P. Lambrecht, N. B. Harris, Z. Feng, D. Daniel Kuczmarski, P. Higley, C. A. Ishimaru, A. Arunakumari, R. G. Barletta and A. K. Vidaver, "Isolation and characterization of endophytic colonizing bacteria from agronomic crops and prairie plants," Appl. Environ. Microbiol. 68(5): 2198-2208, 2002.

[23] K. Tamura, D. Peterson, N. Peterson, G Stecher, M. Nei, and S. Kumar, "MEGA5: Molecular Evolutionary Genetics Analysis using Maximum Likehood, Evolutionary Distance and Maximum Parsimony Methods,” Mol. Biol. Evol., vol. 28, pp: $2731-2739,2011$.

[24] M.K. Halushka, J.B. Fan, K. Bentley, L. Hsie, N. Shen, A. Weder, R. Cooper, R. Lipshutz, and A. Charavarti, "P atterns of singlenucleotide polymorphisms in candidate genes for blood-pressure homestasis," Nat. Genet., vol.22(3), pp:239-247, 1999.

[25] N.T.Thu Ha, H.T. Toan, C.N.Diep, "Isolation anf characrterization of endophytic bacteria in several forage grass cultivars," J. Biotechnology., vol. 7(2), pp:241-250, 2009.

[26] C. N. Diep, N. T. X. My, and V. T. P. Nhu, "Isolation and charcaterization of endophytic bacteria in soybean root nodules," World Journal of Pharmacy and Pharmaceutical Science. vol.5(6), pp;222-241, 2016.

[27] G.A. Watterson, "On the number of segregation sites in general models without recobination," Theor. Pop. Biol., vol. 7, pp. 256-276, 1975.

[28] J. Rozas, and R. Rozas, "DnaSP version 4.1: an integrated program for molecular population genetics and molecular evolution analysis," Bioinformatics, vol. 15, pp: 174-175, 2005.

[29] S. Narula, R.C. Anand, and S.S. Dudeja, "Beneficial traits of endophytic bacteria from field pea nodules and plant growth promotion of field pea," J. Food Legume, vol. 26(3-4), pp:73-79, 2013.

[30] Y.M.Bai , F. D'-Aoust, D.L.Smith and B.T. Driscoll, "Isolation of plant-growth-promoting Bacillus strains from soybean root nodules," Can J Microbiol, vol. 48, pp:230-238, 2002.

[31] M. Senthikumar, V. Govindasamy, and K. Annapurna, "Biocontrol potential of soybean bacterial endophytes againstchar-coal rot fungus Rhizoctonia bataticola," Curr Microbiol., vol. 58, pp:288-293, 2008. 